\title{
Laparoscopic cortical-sparing adrenal surgery in pheochromocytomas associated with hereditary neoplasia syndromes
}

\author{
Kornelia Hasse-Lazar ${ }^{1}$, Marcin Zeman ${ }^{(12}{ }^{2}$, Agnieszka Kotecka-Blicharz ${ }^{(1)}$, Marta Legutko ${ }^{1}$, \\ Agnieszka Czarniecka ${ }^{2}$, Barbara Michalik², Aleksandra Król', Piotr Szymański' , Agnieszka Pawlaczek³, \\ Małgorzata Oczko-Wojciechowska ${ }^{3}$, Maciej Grajek², Tomasz Gawlik¹, Daria Handkiewicz-Junak¹, \\ Barbara Jarząb \\ ${ }^{1}$ Department of Nuclear Medicine and Endocrine Oncology, Maria Sklodowska-Curie National Research Institute of Oncology, \\ Gliwice Branch, Poland \\ ${ }^{2}$ The Oncologic and Reconstructive Surgery Clinic, Maria Sklodowska-Curie National Research Institute of Oncology, \\ Gliwice Branch, Poland \\ ${ }^{3}$ Department of Genetic and Molecular Diagnostics of Cancer, M. Sklodowska-Curie National Research Institute of Oncology, \\ Gliwice Branch, Poland
}

\begin{abstract}
Introduction: Pheochromocytomas in hereditary syndromes tend to grow multifocal with adrenal involvement on both sides. Surgical treatment with bilateral adrenalectomy inevitably leads to life-long hormonal dependence, which significantly affects quality of life. The development of minimally invasive adrenal surgery has created a chance to preserve adrenal cortex function in these patients. The aim of the present study was to evaluate the safety of laparoscopic cortical-sparing adrenal surgeries and their efficacy in the prevention of postoperative adrenal insufficiency in patients with hereditary pheochromocytomas.

Material and methods: We retrospectively analysed the medical histories of 10 patients, who underwent 10 laparoscopic cortical sparing adrenal surgeries from January 2015 to January 2019 in our centre. The decision to perform sparing surgery was based on preoperative diagnosis of hereditary syndrome in line with the result of DNA analysis or its diagnosis based on the clinical appearance. All surgeries were performed laparoscopically from transperitoneal access in the lateral decubitus position, with preserving $1 / 3-1 / 4$ adrenal tissue. The sufficiency of remnant adrenal tissue was assessed in all patients. The median time of follow-up was three years (ranged 0.5-4 years). Results: No intraoperative complications were observed. One case of acute heart failure was the only early postoperative adverse event. There were no late postoperative complications and no local recurrences observed. In one out of three patients undergoing sparing surgery as a second procedure after former total adrenalectomy, adrenal cortex failure occurred. In all patients after unilateral surgery or after bilateral surgery performed simultaneously (total adrenalectomy at one side and sparing surgery contralaterally), function of remnant adrenal tissue was preserved.

Conclusions: In hereditary pheochromocytomas, with minimal risk of malignant process, laparoscopic cortical sparing adrenal surgeries are the safe approach and provide the chance to preserve adrenal cortex function. (Endokrynol Pol 2020; 71 (6): 518-523)
\end{abstract}

Key words: pheochromocytoma; adrenal sparing surgery; adrenal insufficiency

\section{Introduction}

Pheochromocytomas originate from the chromaffin tissue of the adrenal gland and have the ability to produce, metabolise, and secrete catecholamines, leading to hypertension and its complications. About $20-30 \%$ of pheochromocytomas are hereditary and develop due to the mutation in one of the susceptibility genes [1]. Considering also paragangliomas - tumours originating from ganglia of the autonomic nervous system - the percentage of hereditary forms may reach $40 \%$, which makes them the most often genetically determined tumours in humans [2-4].
Due to the increasing amount of clinical and molecular data from large multicentre trials, we have more and more extensive knowledge about genotype-phenotype correlations, affecting the extent and periodicity of patient monitoring, malignancy risk estimation, and therapeutic decisions, including the scope of surgical treatment $[1,4-6]$.

The excision of the tumour with complete removal of the surrounding adrenal tissue is the standard method of management in the case of unilateral tumours. Currently, in the case of benign adrenal lesions, laparoscopic techniques are widely accepted, ensuring safe and effective treatment $[7,8]$. Laparoscopic adrenalectomy 
usually provides complete recovery on the one hand, while providing sufficient hormonal supply from the unchanged contralateral adrenal gland on the other [9]. Patients after surgery do not require hormonal substitution, and further management includes monitoring for local recurrence and metastases, which affect about $10-20 \%$ of all patients and may occur even decades after surgery of the primary tumour. It should be stressed that the postoperative histopathological analysis does not provide a clear distinction between benign and malignant tumours. The existing histopathological scales based on the morphological appearance of tumour cells and elements of immunohistochemical testing are inefficient [10].

Regarding bilateral tumours (developing synchronously or metachronously), this classic approach definitely will lead to the development of adrenal insufficiency and the necessity of long-term hormonal substitution with implications for health and survival. This is associated with both drug overdose and hormone deficiency with the most dangerous complication - adrenal crisis [11, 12].

Therefore, with growing experience in minimally invasive surgery, adrenal sparing surgery has started to be implemented in clinical practice. For this procedure, the tumour is excised with a $3-5 \mathrm{~mm}$ margin of healthy tissue while preserving one quarter to one third of the adrenal cortex, which ensures proper hormonal function. However, the oncological safety of such procedures requires preoperative information concerning low risk of malignant processes [13]. At present, adrenal-sparing surgeries (one-sided or bilateral) are performed in patients at high risk of developing a second tumour in the opposite adrenal gland with a predicted mild course of the disease. These criteria are met in RET, VHL, and NF1 mutation carriers [11, 14, 15].

In patients with multiple endocrine neoplasia syndromes type 2A and 2B (MEN2A/MEN2B) developing due to RET mutation, the risk of pheochromocytoma depends on the type of mutation and is the highest in the case of DNA point lesions located in codon 634 and 918 , where it can be as high as $50 \%$. The risk of bilateral disease is equally high (50\%), but the metastatic disease develops very rarely, and according to the existing data it affects less than $5 \%$ of patients $[2,3,5,16]$.

Pheochromocytomas developing in von Hippel Lindau syndrome (VHL) usually appear in fourth or fifth decade of life, but as the first manifestation of the disease may also occur in children. In $40-50 \%$ of patients the tumours are bilateral. Malignant disease develops at a rate as low as in MEN2 syndromes [1-3].

In neurofibromatosis type 1 syndrome (NF1), pheochromocytomas occur rarely, in about $0.1-6 \%$ of patients, and are usually unilateral; however, in $11-25 \%$ of patients, tumours in the contralateral adrenal gland may appear. They are usually benign, and the average age of disclosure is 40 years.

The aim of the present study was to evaluate the safety of laparoscopic cortical-sparing adrenal surgery and its efficacy in the prevention of postoperative adrenal insufficiency in patients with hereditary pheochromocytomas.

\section{Material and methods}

We retrospectively analysed the medical histories of patients who underwent laparoscopic adrenal-sparing surgery due to the diagnosis of hereditary pheochromocytoma in our centre.

The main qualification criterion for the sparing procedure was confirmation of a hereditary tumour with high risk of bilateral occurrence and low risk of malignancy.

In all patients, molecular testing regarding predisposing germinal mutations was performed. The DNA analysis included nine predisposing genes: RET, VHL, SDHB, SDHC, SDHD, SDHA, SD$H A F 2, M A X$, and TMEM 127. All patients provided signed written informed consent for genetic testing.

Management before surgery comprised the use of the selective postsynaptic alpha-1 blocker (doxazosin) at least two weeks before the operation and, if necessary, supplementation of circulating fluids and application of beta blocker in the pre- or perioperative period. The technical feasibility of the laparoscopic sparing procedure was assessed individually by the operating surgeon.

All surgeries were performed laparoscopically from transperitoneal access in the lateral decubitus position. Pneumoperitoneum was generated by Hasson's method. In the right-side adrenalectomies four ports were used in all cases, while on the left side three or four ports were used. The procedures were performed using monopolar coagulation and a harmonic knife. The adrenal gland, in the case of sparing surgery, was incised with the harmonic knife, followed by the use of a local haemostatic agent. One third to one quarter of the adrenal cortex parenchyma was preserved, depending on tumour size and local conditions according to individual surgeon's assessment. The surgical area was drained, and the drain was removed after 1-3 days. Postoperative complications were evaluated on the Clavien-Dildo scale.

In patients after unilateral surgery, with preserved opposite adrenal gland, hormonal substitution was not implemented and laboratory evaluation of corticotropic axis was performed in the first week after surgery.

In patients after sparing surgery of only one remaining adrenal gland, hydrocortisone was routinely recommended in adequate doses. 1-3 months after surgery, the sufficiency of remnant adrenal tissue was assessed. The patients were qualified for further hormonal substitution if the morning serum cortisol concentration was below $5 \mathrm{mcg} / \mathrm{dL}, 24$ hours after the last dose of hydrocortisone. In patients with morning cortisol concentration above $14 \mathrm{mcg} / \mathrm{dl}$, the normal function of adrenal cortex was assumed. In patients with serum cortisol concentration between 5 and $14 \mathrm{mcg} / \mathrm{dL}$, a test with $250 \mathrm{ug}$ of synthetic adrenocorticotropin (Synacthen, Alfasigma) was performed. Serum cortisol concentration was measured 0 , 30 , and 60 minutes after administration of Synacthen. Cortisol values above $16 \mathrm{mcg} / \mathrm{dL}$ at 30 or 60 minutes after stimulation were assumed to be normal.

Follow-up after surgery encompassed arterial pressure monitoring, and annual measurements of 24-hour urine metanephrines with imaging (CT or MRI) carried out once every 12-24 months. In patients with a preserved part of the adrenal cortex after sparing surgery of only one remnant adrenal gland, clinical evaluation of hormonal substitution with exploration of possible adrenal insufficiency symptoms was periodically performed. 
Table 1. Types of surgery in 10 analysed patients

\begin{tabular}{|c|c|c|c|c|c|}
\hline \multicolumn{2}{|c|}{ Inherited syndrome } & \multirow{2}{*}{$\frac{\text { MEN2 }}{3}$} & \multirow{2}{*}{$\frac{\text { VHL }}{1}$} & \multirow{2}{*}{$\frac{\text { NF1 }}{0}$} & \multirow{2}{*}{$\begin{array}{c}\text { Total } \\
4 \\
\end{array}$} \\
\hline \multirow{5}{*}{ 离 } & Unilateral & & & & \\
\hline & Bilateral & 3 & 2 & 1 & 6 \\
\hline & Synchronous & 1 & 2 & 0 & 3 \\
\hline & Metachronous & 2 & 0 & 1 & 3 \\
\hline & Total & 6 & 3 & 1 & 10 \\
\hline
\end{tabular}

MEN 2 - multiple endocrine neoplasia type 2; VHL — von Hippel-Lindau syndrome; NF1 - neurofibromatosis type 1

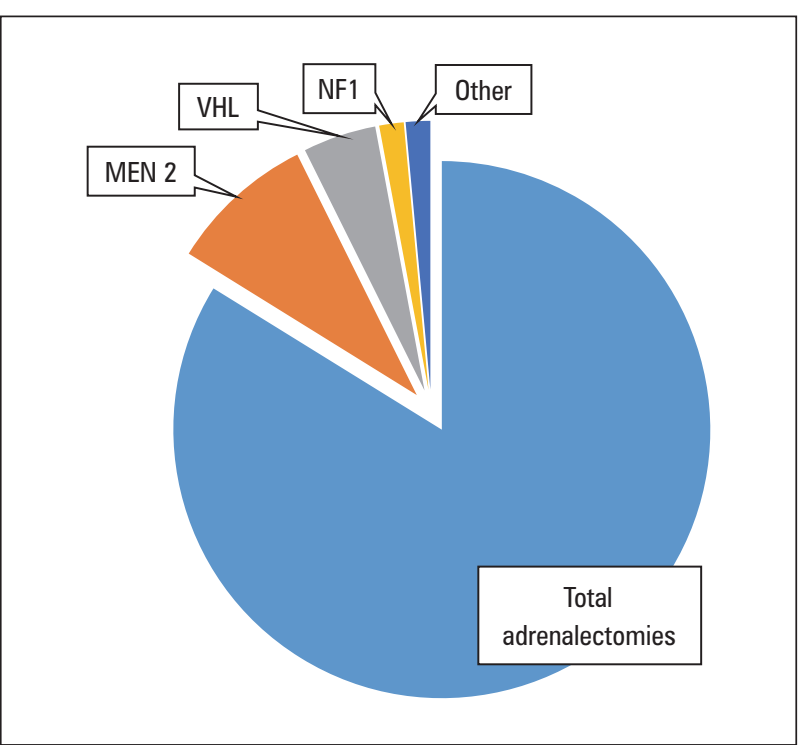

Figure 1. Proportion of laparoscopic adrenal sparing surgeries according to genetic background compared with total adrenalectomies. MEN 2 - multiple endocrine neoplasia type 2; VHL — von Hippel-Lindau syndrome; NF1 — neurofibromatosis type 1; other - without confirmation of hereditary syndrome (not included in this analysis)

\section{Statistical analysis}

Due to limited data, only the age of patients, size of the resected tumour, and time of follow-up were statistically analysed.

\section{Results}

From January 2010 to January 2019, 57 patients underwent 68 laparoscopic adrenalectomies due to the diagnosis of a pheochromocytoma. The present analysis involves 10 of these patients ( 7 females and 3 males), who underwent 10 laparoscopic adrenal sparing surgeries, introduced in 2015 (Tab. 1, Fig. 1).

Based on the medical history, clinical picture, and DNA analysis, MEN2A was diagnosed in four patients, MEN2B in two patients, and VHL syndrome in three. In one patient the diagnosis of hereditary syndrome was based on NF1 phenotypic features (without DNA confirmation). Demographic data of
Table 2. Patient characteristics

\begin{tabular}{lc}
\hline Sex & \\
\hline Male & 3 \\
\hline Female & 7 \\
\hline Median age at first surgery (years) & 31 (range 18-54) \\
\hline Median age at last surgery (years) & 35 (range 23-58) \\
\hline Type of inherited syndrome & \\
\hline MEN2A & 2 \\
\hline MEN2B & 3 \\
\hline VHL & 1 \\
\hline NF1 & 2 \\
\hline
\end{tabular}

MEN 2 - multiple endocrine neoplasia type 2; VHL — von Hippel-Lindau syndrome; NF1 - neurofibromatosis type 1

patients and DNA analysis results are presented in Table 2 .

Four patients were operated on, for the first time in their life, due to a pheochromocytoma located in one adrenal gland, and they underwent unilateral sparing surgery.

In three consecutive patients with synchronous bilateral tumours, also operated on for the first time, the surgical approach included total adrenalectomy on one side and sparing adrenalectomy at the contralateral gland.

The remaining three patients had already undergone laparoscopic unilateral adrenalectomy, and the sparing surgery was the second procedure carried out in their life.

Thus, among patients undergoing bilateral surgery at the same time and among patients undergoing bilateral surgery at time intervals, one of the adrenal glands was completely removed and the second one was partly preserved.

The median age at first operation was 31 years (range 18-54); the median age at second operation was 35 years (range 23-58).

No intraoperative complications were observed. The mean blood loss was $30 \mathrm{ml}$.

Early postoperative adverse events concerned one case of acute heart failure with pulmonary oedema (grade 4A on the Clavien-Dindo scale) requiring support of intensive care unit.

Histopathological analysis confirmed preoperative suspicion of pheochromocytoma in all patients, with free microscopic margins $1-5 \mathrm{~mm}$ wide. The median size of lesions was $15 \mathrm{~mm}$ (range 10-28 mm), which was consistent with preoperative CT. No multiple tumours were observed in the investigated material.

In none of the patients, after unilateral surgery,with the second adrenal gland left intact or after bilateral surgery performed simultaneously (total adrenalectomy at 


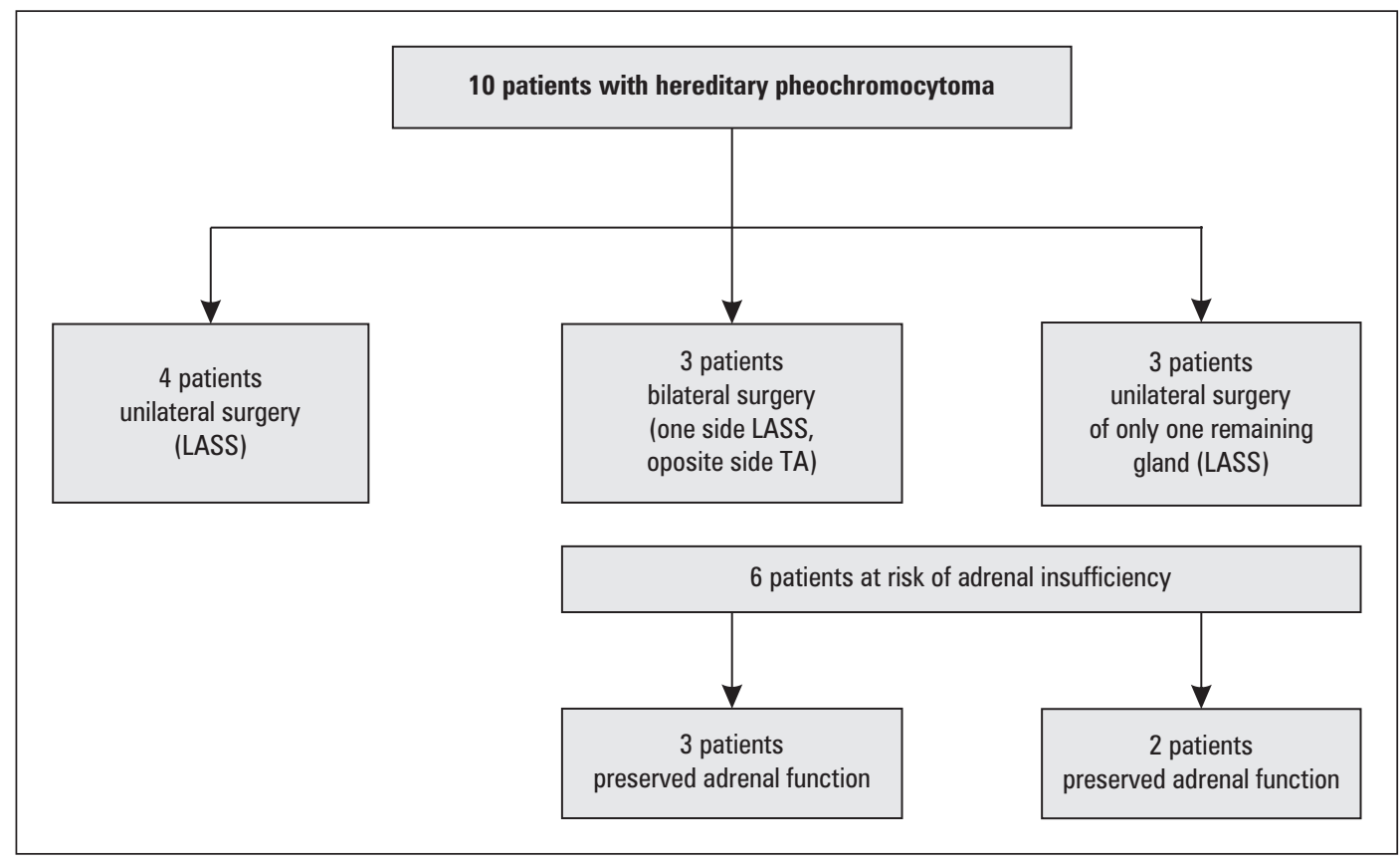

Figure 2. Prevention of adrenal insufficiency according to type of surgery. LASS — laparoscopic adrenal sparing surgery; TA - laparoscopic total adrenalectomy

Table 3. Synacthen test results in six patients after bilateral adrenalectomy

\begin{tabular}{lcccc}
\hline Patient & $\begin{array}{c}\text { Serum } \\
\text { cortisol 0' } \\
{[\mathbf{m c g} / \mathbf{d L}]}\end{array}$ & $\begin{array}{c}\text { Serum } \\
\text { cortisol 30' } \\
{[\mathbf{m c g} / \mathbf{d L}]}\end{array}$ & $\begin{array}{c}\text { Serum } \\
\text { cortisol 60' } \\
{[\mathbf{m c g} / \mathbf{d L}]}\end{array}$ & $\begin{array}{c}\text { Steroid } \\
\text { dependency }\end{array}$ \\
\hline 1 & 10.7 & 11 & 10 & Yes \\
\hline 2 & 10 & 17.8 & 21.3 & No \\
\hline 3 & 8.2 & 12.8 & 16.3 & No \\
\hline 4 & 6.4 & 16.7 & 17.8 & No \\
\hline 5 & 9 & 20.7 & 21.2 & No \\
\hline 6 & 12 & 18 & 19.4 & No \\
\hline
\end{tabular}

one side and sparing surgery contralaterally), as well as in two out of three patients undergoing bilateral surgery in two consecutive steps, no adrenal insufficiency was observed and no hormonal substitution was necessary (Fig. 2).

In one of the three patients undergoing sparing surgery as a second procedure after former total adrenalectomy, adrenal cortex failure requiring continuous glucocorticoid and mineralocorticoid substitution appeared.

The efficacy of sparing surgery in the prevention of adrenal insufficiency according to the type of surgical procedure is illustrated in Figure 2.

The results of the Synacthen test are shown in Table 3.

The median time of follow-up after surgery was three years, ranging from 0.5 to 4 years.

There were no late postoperative complications and no local recurrences observed.
Among the patients with preserved adrenal function after surgery, no late failure occurred.

\section{Discussion}

Despite the lack of randomised clinical trials, sparing adrenalectomies are becoming a routine practice in departments experienced in adrenal surgery [17-19]. This procedure is often technically more demanding than total adrenalectomy, mainly due to haemostasis aspects. However, the frequency of complications after adrenal sparing surgeries is not higher than for total adrenalectomies [5]. This is confirmed by our results, because no intraoperative complications were observed. The only one severe postoperative complication was noticed, which was pulmonary oedema in a 20 -year-old female patient, resulting from hormonal activity of the tumour rather than strictly from surgical causes.

The option of sparing surgery is limited by several factors. The main limitation is the size of the tumour and accordingly the volume of the gland that can be preserved. Therefore, in sporadic tumours, being disclosed late and thus of a greater dimensions, such a surgical approach may be impossible. The sparing procedure is more often recommended in hereditary tumours, usually detected earlier due to active surveillance of mutation carriers, and thereby of a smaller size at the time of diagnosis [2, 3]. In our analysis, in all of our patients, the hereditary syndrome had already been diagnosed prior the surgery and pheochromocytoma was revealed due to annual monitoring. Thus, the size of the tumour 
ranged from 10 to $28 \mathrm{~mm}$ (median $15 \mathrm{~mm}$ ), yielded the possibility of sparing surgery.

Another issue is the localisation of the tumour, which often determines the possibility of preserving sufficient vascularisation of remnant adrenal tissue. Providing at least one active adrenal artery and adrenal vein is considered optimal. However, even in cases when it seems to be impossible, one may consider an attempt of a sparing procedure due to a well-developed network of additional vessels, which is particularly noticeable in pheochromocytomas [5].

Moreover, the impossibility to save the fragment of the gland may result from multiplicity of lesions, which is characteristic for hereditary syndromes. Furthermore, it raises another question about the possibility of new tumours occurring in the remnant adrenal tissue. However, as demonstrated in a large retrospective study by Castinetti et al. [5], the risk of local recurrence is low $(3 \%)$ and does not differ significantly from such a risk due to complete gland removal. In our material, noting relatively short follow-up (median three years), we did not observe any recurrences. Nonetheless, the constant monitoring for subsequent pheochromocytoma is paramount, not only in the opposite adrenal gland but also in the operated area. Even in patients after bilateral total adrenalectomy, such risk exists, and in the analysis by Castinetti et al. it was estimated at 2\% [5].

It is assumed that properly performed adrenal sparing surgery involves excision of the tumour with a 3-7 mm margin of healthy tissue, but in the absence of obvious preoperative evaluation, such an approach may be risky in the case of tumours suspected of a malignant process. Therefore, sparing surgery may be not optimal in patients with sporadic tumours of large size $(>5 \mathrm{~cm})$ and contraindicated in tumours infiltrating adjacent tissues or in SDHB mutation carriers with high risk of malignant process. In such cases, complete adrenalectomies or adrenalectomies with lymphadenectomy are recommended, preferably performed through open approach. Sparing surgeries are reserved for patients with low risk of malignant tumours, such as in RET, VHL, and NF1 gene mutation carriers $[1,6,14,19]$. This indicates the necessity of DNA tests prior to the surgery to resolve the above doubts. If possible, obtaining a detailed medical history of the patient (presence of hereditary syndrome attributes) and family members may direct further therapeutic decisions [10, 15, 20]. In our study preoperative DNA analysis and clinical assessment allowed us to exclude the possibility of tumours with high risk of malignancy in all patients.

When performing bilateral surgery, it is possible to preserve the adrenal gland tissue on both sides or only on one side. In our material, all sparing operations were performed on one side. It is questionable whether to perform a sparing procedure in cases of one-side tumour [10]. However, it seems that such management may increase the chance of saving the function of the adrenal cortex if the adrenalectomy on the opposite side is necessary in the future. There is always some risk that if the subsequent tumour appears in the contralateral gland, the sparing surgery may not be feasible or the preserved part of adrenal cortex may not maintain its function (as was observed in one of our patients).

Preventing steroid dependence and its consequences, in patients with high risk of bilateral pheochromocytoma, was the main reason for introducing partial adrenalectomy [5, 19]. Neumann et al. [20], in a large multicentre study involving patients with hereditary pheochromocytomas after bilateral total adrenalectomy, demonstrated increased risk of adrenal crisis and/or iatrogenic Cushing syndrome in as many as one third of patients, pointing out that the retrospective nature of the study might underestimate the rate of reported complications. The issue is of particular importance because it concerns the group of patients in whom the pheochromocytoma is diagnosed and its treatment is carried out significantly earlier than in the group of patients with sporadic tumours $[1,21]$. In our material, the average age at the first operation was 35 years, whereas the youngest patient operated on bilaterally was 18 years old. While pheochromocytoma disclosure in MEN2A syndrome usually occurs during teenage years, in VHL syndrome it may also affect younger children [12, 21]. Taking into account the medical progress in the prevention and treatment of other components of the aforementioned hereditary syndromes (e.g. prophylactic surgery of medullary thyroid carcinoma), the decrease in quality of life due to steroid substitution, becomes essential in the surveillance of these patients. In Neumann et al. study the risk of adrenal insufficiency in patients after sparing adrenal surgery was estimated at $23.5 \%$, and in Castinetti et al. analysis it was $43 \%[5,19]$. In our study, we managed to maintain normal adrenal function in five out of six patients who were operated on both sides (83\%), and no functional deterioration of remnant adrenal tissue was observed during the subsequent years of observation. However, in the interpretation of the study results, the small size of the examined group and the short time of follow-up should be taken into account.

\section{Conclusions}

Laparoscopic cortical-sparing adrenal surgeries are a safe approach and involve little risk of postoperative complications. In the majority of cases, their performance allows the preservation of the hormonally efficient part of adrenal cortex. Currently, they are carried 
out in patients at risk of bilateral pheochromocytomas development, with minimal risk of malignancy. The type of surgery approach should be decided by both surgeon and endocrinologist.

\section{Authors' statement}

K.H.L. and M.Z. contributed equally.

\section{References}

1. Rossitti HM, Söderkvist P, Gimm O. Extent of surgery for phaeochromocytomas in the genomic era. Br J Surg. 2018; 105(2): e84-e98, doi: 10.1002/bjs.10744, indexed in Pubmed: 29341163

2. Favier J, Amar L, Gimenez-Roqueplo AP. Paraganglioma and phaeochromocytoma: from genetics to personalized medicine. Nat Rev Endocrinol. 2015; 11(2): 101-111, doi: 10.1038/nrendo.2014.188, indexed in Pubmed: 25385035.

3. Dahia PLM. Pheochromocytoma and paraganglioma pathogenesis: learning from genetic heterogeneity. Nat Rev Cancer. 2014; 14(2) 108-119, doi: 10.1038/nrc3648, indexed in Pubmed: 24442145.

4. Bausch B, Schiavi F, Ni Y, et al. European-American-Asian Pheochromocytoma-Paraganglioma Registry Study Group. Clinical Characterization of the Pheochromocytoma and Paraganglioma Susceptibility Genes SDHA, TMEM127, MAX, and SDHAF2 for Gene-Informed Prevention. JAMA Oncol. 2017; 3(9): 1204-1212, doi: 10.1001/jamaoncol.2017.0223, indexed in Pubmed: 28384794.

5. Neumann HPH, Tsoy U, Bancos I, et al. International Bilateral-Pheochromocytoma-Registry Group. Outcomes of adrenal-sparing surgery or total adrenalectomy in phaeochromocytoma associated with multiple endocrine neoplasia type 2: an internationa retrospective population-based study. Lancet Oncol, 2014; 15(6): 648-655, doi: 10.1016/S1470-2045(14)70154-8, indexed in Pubmed: 24745698 .

6. Kotecka-Blicharz A, Hasse-Lazar K, Jurecka-Lubieniecka B, et al. Occurrence of phaeochromocytoma tumours in RET mutation carriers - a single-centre study. Endokrynol Pol. 2016; 67(1): 54-58, doi: 10.5603/EP.2016.0008, indexed in Pubmed: 26884116.

7. Pogorzelski R, Toutounchi S, Ambroziak U, et al. Effectiveness of unilateral laparoscopic adrenalectomy in ACTH-independent hypercortisolaemia and subclinical Cushing's syndrome - a retrospective study on a large cohort. Endokrynol Pol. 2018; 69(4): 411-415, doi: 10.5603/EP.a2018.0039, indexed in Pubmed: 29952409.

8. Kozłowski T, Rydzewska-Rosolowska A, Myśliwiec J, et al. The impac of laparoscopic adrenalectomy on renal function. Results of a prospective randomised clinical trial. Endokrynol Pol. 2019; 70(5): 409-416, doi: 10.5603/EPa2019.0029, indexed in Pubmed: 31274187.
9. Bednarczuk T, Bolanowski M, Sworczak $\mathrm{K}$, et al. Adrenal incidentaloma in adults - management recommendations by the Polish Society of Endocrinology. Endokrynol Pol. 2016; 67(2): 234-258, doi: 10.5603/EP.a2016.0039, indexed in Pubmed: 27082051.

10. Lenders JWM, Duh QY, Eisenhofer G, et al. Endocrine Society. Pheochromocytoma and paraganglioma: an endocrine society clinical practice guideline. J Clin Endocrinol Metab. 2014; 99(6): 1915-1942, doi: 10.1210/jc.2014-1498, indexed in Pubmed: 24893135.

11. Castinetti F, Taieb D, Henry JF, et al. Managment of endocrine Disease:Outcome of adrenal sparing surgery in heritable pheochromocytoma. Eur J Endocrinol. 2016; 174(1): R9-18, doi: 10.1530/EJE-15-0549, indexed in Pubmed: 26297495

12. Volkin D, Yerram N, Ahmed F, et al. Partial adrenalectomy minimizes the need for long-term hormone replacement in pediatric patients with pheochromocytoma and von Hippel-Lindau syndrome. J Pediatr Surg 2012; 47(11): 2077-2082, doi: 10.1016/j.jpedsurg.2012.07.003, indexed in Pubmed: 23164001.

13. Otto M, Dzwonkowski J. Adrenal-preserving surgery of adrenal tumours. Endokrynol Pol. 2015; 66(1): 80-96, doi: 10.5603/EP.2015.0012, indexed in Pubmed: 25754286.

14. Biteman BR, Randall JA, Brody F. Laparoscopic bilateral cortical-sparing adrenalectomy for pheochromocytoma. Surg Endosc. 2016; 30(12): 5622-5623, doi: 10.1007/s00464-016-4919-5, indexed in Pubmed: 27177950

15. Nockel P, El Lakis M, Gaitanidis A et al. Preoperative genetic testing in pheochromocytomas and paragangliomas influences the surgical approach and the extent of adrenal surgery. Surgery. 2018; 163(1): 191-196 doi: 10.1016/j.surg.2017.05.025, indexed in Pubmed: 29126554.

16. Machens A, Dralle $\mathrm{H}$. Advances in risk-oriented surgery for multiple endocrine neoplasia type 2. Endocr Relat Cancer. 2018; 25(2): T41-T52, doi: 10.1530/ERC-17-0202, indexed in Pubmed: 28883084.

17. Cavallaro G, Letizia C, Polistena A, et al Laparoscopic adrenal-sparing surgery: personal experience, review on technical aspects. Updates Surg. 2011; 63(1): 35-38, doi: 10.1007/s13304-011-0052-0, indexed in Pubmed: 21312015

18. Toutounchi S, Pogorzelski R, Wołoszko T, et al. Adrenal-sparing surgery for a hormonally active tumor - a single-center experience. Endokryno Pol. 2020; Ahead of print, doi: 10.5603/ep.a2020.0033, indexed in Pubmed: 33140380.

19. Lee JE, Curley SA, Gagel RF, et al. Cortical-sparing adrenalectomy for patients with bilateral pheochromocytoma. Surgery. 1996; 120(6): 1064-70; discussion 1070, doi: 10.1016/s0039-6060(96)80056-0, indexed in Pubmed: 8957496

20. Neumann HPH, Tsoy U, Bancos I, et al. International Bilateral-Pheochromocytoma-Registry Group. Comparison of Pheochromocytoma-Specific Morbidity and Mortality Among Adults With Bilateral Pheochromocytomas Undergoing Total Adrenalectomy vs Cortical-Sparing Adrenalectomy. JAMA Netw Open. 2019; 2(8): e198898, doi: 10.1001/jamanetworkopen.2019.8898, indexed in Pubmed: 31397861.

21. Fallon SC, Feig D, Lopez ME, et al. The utility of cortical-sparing adrenalectomy in pheochromocytomas associated with genetic syndromes. J Pediatr Surg. 2013; 48(6): 1422-1425, doi: 10.1016/j.jpedsurg.2013.04.001, indexed in Pubmed: 23845641. 